\title{
Comparative Study on Carcass Traits, Meat Quality and Taste in Broiler, Broiler Breeder and Aseel Chickens
}

\section{-Author(s)}

\begin{tabular}{|c|c|}
\hline $\operatorname{an} U^{\prime}$ & s://orcid.org/0000-0002-7871-1369 \\
\hline 'ssain J' & nttps://orcid.org/0000-0002-4837-375X \\
\hline Mahmud $A^{\prime}$ & https://orcid.org/0000-0002-2106-4113 \\
\hline halique $\mathrm{A}^{\prime \prime}$ & https://orcid.org/0000-0002-5960-025X \\
\hline$d S^{\prime}$ & ://orcid.org/0000-000 \\
\hline adar IH"I & orcid.org/0000-0001-7352-9712 \\
\hline Usman $\mathrm{M}^{\prime}$ & ps://orcid.org/0000-0002-3128-3969 \\
\hline Jaspal MH"' & https://orcid.org/0000-0001-7163-1369 \\
\hline hmad S" & https://orcid.org/0000-0002-0390-3083 \\
\hline
\end{tabular}

Department of Poultry Production, Faculty of Animal Production and Technology, University of Veterinary and Animal Sciences, Lahore, 54000 Pakistan.

" Department of Animal Nutrition, Faculty of Animal Production and Technology, University of Veterinary and Animal Sciences, Lahore, 54000, Pakistan.

III Department of Meat Science and Technology, Faculty of Animal Production and Technology, University of Veterinary and Animal Sciences, Lahore, 54000, Pakistan.

\section{ABSTRACT}

Present study evaluated carcass and meat quality attributes of broilers, broiler breeders and Aseel chickens. Sixty birds from each chicken genotype (broiler, broiler breeder and Aseel chickens; fivewk-old broilers and 60-wk-old Aseel chickens and broiler breeders) were evaluated. Birds were maintained under similar environment for 21 days, and then slaughtered to analyze their carcass qualitative and organoleptic characteristics. No carcass yield differences were detected among genotypes. Aseel chickens had heavier neck, followed by broiler breeder and broiler $(p<0.0001)$. Higher liver $(p<0.0001)$, intestine $(p<0.0001)$, ribs and back $(p=0.0014)$ yields were obtained in broilers than in broiler breeders and Aseel chickens. Females showed higher gizzard $(p=0.0107)$ and intestine $(p=0.0170)$ yield than males, which presented higher carcass $(p=0.0023)$, thigh $(p=0.0039)$, drumstick $(p<0.0001)$, neck $(p=0.0003)$ and heart $(p=0.0139)$ yields. Broiler meat was lighter $(p<0.0001)$ whereas Broiler breeder meat were yellower $(p<0.0001)$ and redder $(p<0.0001)$, ultimate-pH was lower $(p=0.0001)$ for broiler and Aseel meat. Female meat was yellower $(p<0.0001)$ and reddish ( $p=0.0482$ ). Aseel breast meatscored lower for flavor $(p=0.0121)$, juiciness ( $p=0.0178)$ and tenderness $(p=0.0477)$ compared with broiler breeders and broilers, although no differences among genotypes were detected for color, aroma, taste, and acceptability, whereas for thigh meat, Aseel chickens received lower color $(p=0.0344)$ and acceptability $(p=0.0398)$ scores. Interaction effect were significant for carcass, meat quality and sensory evaluation. Carcass characteristics of broilers were comparable with Aseel chickens, while broiler breeder showed better meat quality traits. Broiler and broiler breeder meat scored higher for sensory evaluation. Male birds had higher carcass yield and better meat quality traits compared with females. It is concluded that meat quality attributes vary among the three chicken genotypes.

\section{INTRODUCTION}

World population has continuously grown, leading to increased demand for animal proteins. Poultry meat and eggs, as high-quality animal proteins, are important sources for sustaining health and nutrition of human beings (Shahzad et al., 2011). According to World Health Organization (WHO), $27 \mathrm{~g}$ of animal protein are required per individual on daily basis; however, in Pakistan, this figure is only 17 $\mathrm{g}$ per person per day. Almost $66 \%$ of the population in Pakistan is animal protein-deficient, which may affect the overall health status of the population and as is a big question mark as to the food safety and security in the country (Memon, 2012).

Pakistan poultry sector is playing active role to overcome the gap between demand and supply of animal proteins. According to the Economic Survey (2016-17), the contribution of commercial and rural 
poultry sectors in the meat production has been about $1,054.46$ and 115.24 million metric tons, respectively.

The quality of meat is measured in terms of the major chemical components such as proteins, fats, carbohydrates, minerals and fatty acid contents (Pearson \& Gillet, 1996). Several factors such as genetics, age, live weight and sex have been shown to affect poultry meat yield, its composition and overall quality (Young et al., 2000).

In the recent scenario, consumers have shown a strong interest in the overall nutritional values of food, as well as the role played by specific diets in healthy lifestyle (Karakök et al., 2008). Local farmers and chicken meat consumers have also shown interest in the native germplasm because of its unique characteristics. Indigenous breeds are high in protein, particularly, enriched with essential amino acids and low in calories (Bell \& Weaver, 2002). Generally, consumers prefer indigenous meat because of high fibrous and tasty flavor (Jaturasitha et al., 2002). Muscle fiber type and size also affect meat quality traits and are determined by genotype (Klont et al., 1998). Aseel chicken meat is also famous for its texture, shear force value, large amount of connective tissues and overall acceptability. Because of its high exercise and fighting behaviors, Aseel chickens also present low abdominal fat (Rajkumar et al., 2016).

Along with commercial broilers, a significant number of broiler breeders (both male and female) is also marketed as a meat source in Pakistan at its culling/terminal stage. According to Pakistan Poultry Association (PPA, 2016), about 14 million broiler breeder females were raised in Pakistan and approximately the same number was marketed. However, no qualitative or quantitative data on their meat quality attributes is available.

Meat from different chicken genotypes have different nutritional and qualitative values, but the extent of this variation has not been comprehensively evaluated yet. The main objective of the present study was to capture such variation in the three main chicken meat genotypes marketed in Pakistan: commercial broilers, broiler breeders and Aseel chickens.

\section{MATERIALS AND METHODS}

The present study was conducted at the Department of Poultry Production, University of Veterinary and Animal Sciences (UVAS), Ravi Campus, Pattoki, Pakistan. The study was performed in compliance with the guidelines and code of practices of UVAS, Lahore, Pakistan and ethical approval was obtained.

\section{Experimental birds}

1. Aseel

2. Commercial broilers (Hubbard Classic)

3. Commercial broiler breeders (Hubbard Classic)

\section{Experimental birds}

A total of 60 birds (30 males and 30 females, 10 from each sex of each breed) were studied regarding meat quality attributes at their terminal stage [market age; broilers ( 5 weeks), broiler breeders (60 weeks) and Aseel chickens (60 weeks)]. The Aseel chickens were obtained from breeding flocks maintained at the Indigenous Chicken Genetic Resources Centre (ICGRC) under a semi-intensive system.

The experimental diets were formulated according to the recommendations of NRC (1994) and to the Hubbard Classic broiler and broiler breeder nutrient specifications (Tables 1, 2). At terminal stage, commercial broilers and broiler breeders (Hubbard Classic) were collected from local market, maintained under the same nutritional and environmental conditions for a period of three (3) weeks.

Table 1 - Ingredients and calculated nutrient composition of the experimental diets

\begin{tabular}{lcc}
\hline Feed ingredient (\%) & $\begin{array}{c}\text { Broiler Breeder \& Aseel } \\
\text { Male Diets }\end{array}$ & $\begin{array}{c}\text { Broiler Breeder \& Aseel } \\
\text { Female Diets }\end{array}$ \\
\hline Corn & 39.4 & 42.61 \\
Soybean meal & 10.45 & 15.62 \\
Corn Gluten 60\% & - & 1 \\
Rice Tips & 31 & 19 \\
Wheat bran & 15.8 & 13 \\
\hline Dicalcium phosphate & 0.70 & 1.2 \\
CaCO $_{3}$ & 2.65 & 7.42 \\
\hline DL-Methionine & - & 0.15 \\
Nutrient (\%) & & \\
Crude Protein & 13.13 & 15.04 \\
Metabolizable Energy & 2848 & 2682 \\
(kcal/kg) & & \\
Calcium & 1.09 & 2.81 \\
Phosphorus & 0.22 & 0.34 \\
Lysine & 0.74 & 0.855 \\
Methionine & 0.39 & 0.45
\end{tabular}

\section{Processing}

A total of 60 birds (20 from each genotype and 10 from each sex) were manually slaughtered following Halal method on the same day. After slaughter, birds were manually de-feathered and eviscerated, and the carcasses were then immersed in chilled water for 1 hour. Upon removal from the chiller, carcasses were hanged for dripping and then cut up in different parts for further analyses. Empty carcass, breast, thigh, wing, drumstick, neck, liver, heart, gizzard, intestine, 
Khan U, Hussain J, Mahmud A, Khalique A, Mehmood S, Badar IH, Usman M, Jaspal MH, Ahmad S

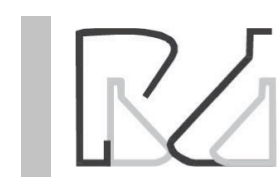

Comparative Study on Carcass Traits, Meat Quality and Taste in Broiler, Broiler Breeder and Aseel Chickens ribs and back were weighed and their yield calculated as a percentage of live weight at slaughter.

Table $\mathbf{2}$ - Ingredients and calculated nutrient composition of the experimental broiler diet.

\begin{tabular}{lccc}
\hline Ingredients & $(\%)$ & Nutrients & \\
\hline Corn & 54.85 & Metabolizable energy (kcal/kg) & 2800 \\
Rice polish & 5.00 & Crude protein (\%) & 20.0 \\
Wheat bran & 3.00 & Fat (\%) & 4.11 \\
Canola meal & 6.05 & Fiber (\%) & 4.31 \\
Rapeseed meal & 4.00 & Calcium (\%) & 0.82 \\
Soybean meal & 16.00 & Available phosphorus (\%) & 0.4 \\
\hline Corn gluten meal & 1.60 & Dig. Lysine (\%) & 1.05 \\
Poultry byproduct meal & 2.00 & Dig. Methionine (\%) & 0.49 \\
Fish meal & 2.50 & Dig. Methionine+cystine (\%) & 0.77 \\
Marble chips & 0.55 & Dig. Arginine (\%) & 1.1 \\
\hline Dicalcium phosphate & 0.53 & Dig. Threonine (\%) & 0.66 \\
Lysine sulphate & 0.48 & Dig. Tryptophan (\%) & 0.18 \\
DL-methionine & 0.18 & Dig. Isoleucine (\%) & 0.68 \\
Threonine & 0.05 & Dig. Valine (\%) & 0.76 \\
Molasses & 2.50 & & \\
Premix* & 0.43 & & \\
Salt & 0.23 & & \\
Phytase & 0.05 & & \\
Rice broken & 0.00 & & \\
\hline Total & 100 & & \\
\hline
\end{tabular}

*Vitamin-mineral premix supplied per $\mathrm{Kg}$ of diet: vitamin A, 11,000 IU; vitamin D3, 2,560 IU; vitamin E, $44 \mathrm{IU}$; vitamin K, $4.2 \mathrm{mg}$; riboflavin, $8.5 \mathrm{mg}$; niacin, $48.5 \mathrm{mg}$; thiamine, $3.5 \mathrm{mg}$; d-pantothenic, $27 \mathrm{mg}$; choline, $150 \mathrm{mg}$; vitamin B12, $33 \mu \mathrm{g}$; copper, $8 \mathrm{mg}$; zinc, $75 \mathrm{mg}$; manganese, $55 \mathrm{mg}$; iodine, $0.35 \mathrm{mg}$; selenium, $0.15 \mathrm{mg}$.

\section{Meat quality}

Approximately 4 hours after slaughter, breast and thigh meat were separated. Meat (breast and thigh) color was measured in duplicate / sample using chromameter (Konica Minolta Chroma Meter CR-41) and lightness, redness and yellowness $\left(L^{*}, a^{*}, b^{*}\right)$ values were recorded. Meat $\mathrm{pH}$ value was measured $24 \mathrm{~h}$ after slaughter (ultimate $\mathrm{pH}$ ) using a $\mathrm{pH}$ meter (Weilheim, WTW GmbH, model WTW-3210, Germany). Meat samples were placed in plastic bag, hung for 24 hours at $8-10^{\circ} \mathrm{C}$, blotted dry, and weighed again to measure drip loss (Honikel, 1987). Meat samples were stored at $5^{\circ} \mathrm{C}$ for 24 hours, after which 2 cylindrical pieces of meat (parallel to the fibers, diameter $12 \mathrm{~mm}$, at least 3 $\mathrm{cm}$ long) were cut from each breast and used for shear force test by using a Warner-Bratzler (TAXT Plus, USA) shear force texture analyzer (Stadig et al., 2016).

\section{Sensory Characteristics}

Sensory panel test on breast and thigh samples was separately performed by roasting the samples without salt or spices (Castellini et al., 2002). The cooked samples were immediately sliced into pieces and was offered to panelists $(n=25)$. For each sensory characteristic, participants were instructed to score the intensity of evaluation on a nine-point hedonic scale (1 for extremely dislike, 2 for dislike very much, 3 for moderately dislike, 4 for slightly dislike, 5 for neither like nor dislike, 6 for slightly like, 7 for moderately like, 8 for like very much and 9 for extremely like). The parameters evaluated included color, aroma, taste, flavor, juiciness, tenderness, and overall acceptability.

\section{Statistical Analysis}

The parameters are presented as least square mean \pm standard error. Prior to analyses, homogeneity of variance was tested and normality of data were verified. A two-way analysis of variance was employed to analyze the data applying the General Linear Model procedure of SAS software (version 9.1, SAS, 20022004). In case of pair-wise comparisons, the TukeyKramer adjustment for multiple comparisons was used. Significant difference was based on $p \leq 0.05$, unless otherwise stated. Following mathematical model was used:

Where,

$$
Y_{i j k}=\mu+G_{i}+S_{j}+\left(G_{i} \times S_{j}\right)+\varepsilon_{i j k}
$$

$Y_{i j k}=$ Observation of dependent variable recorded on $i^{\text {th }}$ genotype of $j^{\text {th }}$ sex

$\mu=$ Population mean

$G_{i}=$ Effect of $i^{\text {th }}$ genotype $(i=1,2,3)$

$S_{i}=$ Effect of $j^{\text {th }} \operatorname{sex}(j=1,2)$

$\mathrm{G}_{\mathrm{i}} \times \mathrm{S}_{\mathrm{i}}=$ Interaction between genotype and sex

$\varepsilon_{\mathrm{ijk}}=$ Residual error of $\mathrm{k}^{\text {th }}$ observation recorded on $\mathrm{ith}^{\text {th }}$ genotype and $j^{\text {th }}$ sex NID $\sim 0, \sigma^{2}$

\section{RESULTS}

\section{Carcass Traits}

The effects of genotype and sex on carcass traits are shown in Table 3.

Broilers and broiler breeders presented heavier breasts than Aseel chickens $(p<0.0001)$. Heavier thighs and drumsticks were determined in Aseel chickens and broiler breeders than in broilers $(p<0.0001)$. Aseel chickens had heavier necks, followed by broiler breeders and broilers $(p<0.0001)$. Higher liver $(p<0.0001)$, intestines $(p<0.0001)$, ribs and back $(p=0.0014)$ weights were measured in broilers and broiler breeders than in Aseel chickens. Heart and gizzard were heavier in broilers and Aseel chickens than in broiler breeders $(p<0.0001)$. Average carcass yield $(p=0.2625)$ and wing yield $(p=0.0808)$ were not different among genotypes. 


\section{Comparative Study on Carcass Traits, Meat Quality and Taste in Broiler, Broiler Breeder and Aseel Chickens}

Table 3 - Carcass traits of Aseel chickens, broiler breeders and broilers, expressed as percentage of live body weight $(n=20)$.

\begin{tabular}{|c|c|c|c|c|c|c|c|}
\hline \multirow[t]{2}{*}{ Yield (\%) } & \multicolumn{3}{|c|}{ Genotype } & \multirow[t]{2}{*}{$p$-value } & \multicolumn{2}{|c|}{ Sex } & \multirow[t]{2}{*}{$p$-value } \\
\hline & Aseel & Broiler Breeder & Broiler & & Male & Female & \\
\hline Carcass & $61.74 \pm 0.65$ & $64.73 \pm 1.39$ & $62.96 \pm 1.19$ & 0.2625 & $64.99 \pm 0.81^{a}$ & $61.04 \pm 0.86^{b}$ & 0.0023 \\
\hline Breast & $13.20 \pm 0.48^{b}$ & $18.67 \pm 0.74^{a}$ & $18.69 \pm 0.72^{a}$ & $<0.0001$ & $16.81 \pm 0.79$ & $16.99 \pm 0.73$ & 0.5051 \\
\hline Thigh & $12.24 \pm 0.46^{a}$ & $11.92 \pm 0.51^{\mathrm{a}}$ & $9.10 \pm 0.28^{b}$ & $<0.0001$ & $11.56 \pm 0.47^{a}$ & $10.35 \pm 0.39^{b}$ & 0.0039 \\
\hline Wing & $6.48 \pm 0.20$ & $5.95 \pm 0.20$ & $5.90 \pm 0.22$ & 0.0808 & $6.13 \pm 0.14$ & $6.08 \pm 0.21$ & 0.5764 \\
\hline Drumstick & $10.46 \pm 0.36^{a}$ & $10.56 \pm 0.43^{a}$ & $8.85 \pm 0.20^{b}$ & $<0.0001$ & $10.60 \pm 0.33^{a}$ & $9.12 \pm 0.18^{b}$ & $<0.0001$ \\
\hline Neck & $3.68 \pm 0.20^{a}$ & $2.97 \pm 0.11^{b}$ & $1.86 \pm 0.10^{c}$ & $<0.0001$ & $2.98 \pm 0.23^{a}$ & $2.58 \pm 0.14^{b}$ & 0.0003 \\
\hline Liver & $1.90 \pm 0.11^{b}$ & $1.64 \pm 0.13^{b}$ & $3.17 \pm 0.25^{a}$ & $<0.0001$ & $2.34 \pm 0.22$ & $2.24 \pm 0.19$ & 0.7893 \\
\hline Heart & $0.66 \pm 0.04^{b}$ & $0.52 \pm 0.02^{c}$ & $0.91 \pm 0.07^{\mathrm{a}}$ & $<0.0001$ & $0.78 \pm 0.06^{a}$ & $0.64 \pm 0.04^{b}$ & 0.0139 \\
\hline Gizzard & $1.73 \pm 0.11^{b}$ & $1.28 \pm 0.10^{c}$ & $2.36 \pm 0.10^{a}$ & $<0.0001$ & $1.75 \pm 0.16^{b}$ & $1.91 \pm 0.06^{a}$ & 0.0107 \\
\hline Intestine & $2.13 \pm 0.12^{c}$ & $2.63 \pm 0.23^{b}$ & $3.77 \pm 0.17^{\mathrm{a}}$ & $<0.0001$ & $2.75 \pm 0.24^{b}$ & $3.03 \pm 0.15^{a}$ & 0.0170 \\
\hline Ribs and back & $19.41 \pm 0.41^{\mathrm{ab}}$ & $17.80 \pm 0.66^{b}$ & $20.91 \pm 0.59^{a}$ & 0.0014 & $19.46 \pm 0.49$ & $19.49 \pm 0.57$ & 0.9173 \\
\hline
\end{tabular}

acc Means followed by different superscripts within a row differ significantly $(p \leq 0.05)$.

Relative to the effect of sex, heavier gizzards $(p=0.0107)$ and intestines $(p=0.0170)$ were obtained in females than in males. On the other hand, males showed higher carcass $(p=0.0023)$, and thigh $(p=0.0039)$, drumstick $(p<0.0001)$, neck $(p=0.0003)$ and heart $(p=0.0139)$ yields than females. No differences in breast, wing, liver, ribs and back yields were detected between sexes (Table 3).

There was a significant interaction $(p \leq 0.05)$ between genotype and sex for all the carcass traits (Table 4). Broiler breeder males presented higher carcass yield than females of all genotypes, whereas Aseel and broiler males show intermediate values $(p=0.0175)$. Higher breast yield was obtained in both male and female broilers and broiler breeders relative to Aseel chickens of both sexes $(p<0.0001)$. Higher thigh yield was observed in Aseel male as compared to Aseel and Broiler breeder females, however, broiler breeder male revealed intermediate values. Furthermore, broiler male and female represent lower value $(p<0.0001)$. Wing percentage was higher in Aseel female compared to Broiler breeder female whereas rest of the treatment

Table 4 - Genotype $\times$ sex interactions for carcass traits of Aseel chickens, broiler breeders and broilers, expressed as percentage of live body weight $(n=10)$.

\begin{tabular}{|c|c|c|c|c|c|c|c|}
\hline \multirow[t]{2}{*}{ Yield \% } & \multicolumn{2}{|c|}{ Aseel } & \multicolumn{2}{|c|}{ Broiler Breeder } & \multicolumn{2}{|c|}{ Broiler } & \multirow[t]{2}{*}{$p$-value } \\
\hline & Male & Female & Male & Female & Male & Female & \\
\hline Carcass & $63.19 \pm 0.92^{\mathrm{ab}}$ & $60.46 \pm 0.68^{b}$ & $67.25 \pm 1.90^{a}$ & $61.36 \pm 0.95^{b}$ & $64.36 \pm 0.91^{\mathrm{ab}}$ & $61.38 \pm 2.26^{b}$ & 0.0175 \\
\hline Breast & $12.53 \pm 0.52^{b}$ & $13.79 \pm 0.74^{b}$ & $18.89 \pm 1.27^{a}$ & $18.37 \pm 0.58^{a}$ & $18.30 \pm 0.94^{a}$ & $19.14 \pm 1.15^{a}$ & $<0.0001$ \\
\hline Thigh & $13.42 \pm 0.73^{a}$ & $11.20 \pm 0.23^{b}$ & $12.62 \pm 0.51^{a b}$ & $10.98 \pm 0.90^{b}$ & $9.17 \pm 0.18^{c}$ & $9.03 \pm 0.58^{c}$ & $<0.0001$ \\
\hline Wing & $6.22 \pm 0.25^{\mathrm{ab}}$ & $6.72 \pm 0.29^{a}$ & $6.39 \pm 0.17^{a b}$ & $5.37 \pm 0.27^{b}$ & $5.84 \pm 0.29^{a b}$ & $5.97 \pm 0.36^{\mathrm{ab}}$ & 0.0358 \\
\hline Drumstick & $11.64 \pm 0.38^{a}$ & $9.43 \pm 0.24^{b}$ & $11.66 \pm 0.37^{a}$ & $9.08 \pm 0.34^{b}$ & $8.86 \pm 0.24^{b}$ & $8.84 \pm 0.34^{b}$ & 0.0004 \\
\hline Neck & $4.36 \pm 0.14^{a}$ & $3.08 \pm 0.18^{b}$ & $3.15 \pm 0.14^{b}$ & $2.74 \pm 0.15^{b}$ & $1.76 \pm 0.13^{c}$ & $1.96 \pm 0.14^{c}$ & $<0.0001$ \\
\hline Liver & $1.76 \pm 0.13^{c}$ & $2.02 \pm 0.17^{b c}$ & $1.46 \pm 0.07 c$ & $1.89 \pm 0.27^{b c}$ & $3.58 \pm 0.24^{a}$ & $2.72 \pm 0.42^{b}$ & 0.0245 \\
\hline Heart & $0.66 \pm 0.03^{b}$ & $0.65 \pm 0.08^{b}$ & $0.49 \pm 0.03^{b}$ & $0.57 \pm 0.03^{b}$ & $1.13 \pm 0.07^{a}$ & $0.68 \pm 0.07^{b}$ & $<0.0001$ \\
\hline Gizzard & $1.41 \pm 0.12^{c}$ & $2.01 \pm 0.11^{b}$ & $1.00 \pm 0.03^{d}$ & $1.65 \pm 0.12^{c}$ & $2.67 \pm 0.08^{a}$ & $2.02 \pm 0.05^{b}$ & $<0.0001$ \\
\hline Intestine & $2.01 \pm 0.17^{b}$ & $2.24 \pm 0.16^{b}$ & $2.00 \pm 0.17^{b}$ & $3.46 \pm 0.13^{\mathrm{a}}$ & $4.00 \pm 0.29^{a}$ & $3.50 \pm 0.13^{a}$ & $<0.0001$ \\
\hline Ribs and back & $18.78 \pm 0.43^{a b}$ & $19.95 \pm 0.63^{a b}$ & $18.27 \pm 0.81^{a b}$ & $17.18 \pm 1.12^{b}$ & $21.05 \pm 0.83^{a}$ & $20.76 \pm 0.89^{a}$ & 0.0122 \\
\hline
\end{tabular}

${ }^{a-d}$ Means followed by different superscripts within a row differ significantly $(p \leq 0.05)$.

groups showed intermediate values $(p=0.0358)$. Aseel and Broiler breeder male showed higher drumstick percentage as compared to the rest of the treatments $(p=0.0004)$. Aseel male had higher neck followed by Aseel female and both sexes of broiler breeder and broiler $(p<0.0001)$. Liver percentage was higher in broiler male than female. Furthermore, Aseel and Broiler breeder female showed intermediate values whereas their male counterparts showed lower values $(p=0.0245)$. Broiler male had higher percentage of heart as compared to the rest of the treatments $(p<0.0001)$. Higher gizzard percentage was observed in broiler male followed by Broiler and Aseel female, Aseel male and broiler breeder male and female $(p<0.0001)$. Intestinal weight was higher in broiler breeder female and broiler of both sexes as compared to rest of the treatments $(p<0.0001)$. Ribs and back percentage were higher in broiler male and female as compared to broiler breeder female whereas male Broiler breeder and Aseel of both sexes presented intermediate values $(p=0.0122)$. 


\section{Comparative Study on Carcass Traits, Meat Quality and Taste in Broiler, Broiler Breeder and Aseel Chickens}

\section{Meat quality}

Breast meat color differed among genotypes (Table 5 ). The meat of Aseel chickens and broiler breeders was darker $(p<0.0001)$ than that of broilers. Broiler breeder meat were yellower $(p<0.0001)$ and redder $(p<0.0001)$ than those of Aseel chickens and broilers. Lower ultimate $\mathrm{pH}(p=0.0001)$ was determined in the meat of broilers and Aseel chickens than in broiler breeders. No significant differences in drip loss $(p=0.0976)$ or shear force $(p=0.0998)$ were found among genotypes.
The breast meat of females was yellower $(p<0.0001)$ and redder $(p=0.0482)$ compared to males. There were no differences in meat lightness $(p=0.2351)$, ultimate $\mathrm{pH}(p=0.4278)$, drip loss $(p=0.7821)$ or shear force $(p=0.4506)$ between sexes (Table 5$)$.

Meat of Broiler male and female were lighter $(p<0.0001)$ than that of Aseel and Broiler breeder. Broiler breeder meat of both sexes were more reddish $(p<0.0001)$ as compared to Aseel and Broiler. Similarly, broiler breeder meat of both sexes was yellower

Table 5 - Meat quality attributes Aseel chickens, broiler breeders and broilers.

\begin{tabular}{|c|c|c|c|c|c|c|c|}
\hline \multirow[t]{2}{*}{ Parameter } & \multicolumn{3}{|c|}{ Genotype } & \multirow[t]{2}{*}{$p$-value } & \multicolumn{2}{|c|}{ Sex } & \multirow[t]{2}{*}{$p$-value } \\
\hline & Aseel & Broiler Breeder & Broiler & & Male & Female & \\
\hline$L^{*}$ & $53.66 \pm 0.68^{b}$ & $55.69 \pm 1.21^{b}$ & $62.57 \pm 0.44^{a}$ & $<0.0001$ & $57.98 \pm 1.04$ & $56.18 \pm 1.07$ & 0.2351 \\
\hline$a^{*}$ & $11.20 \pm 0.38^{b}$ & $13.24 \pm 0.26^{a}$ & $9.81 \pm 0.29$ & $<0.0001$ & $11.15 \pm 0.40^{b}$ & $11.94 \pm 0.37^{a}$ & 0.0482 \\
\hline$b^{*}$ & $6.14 \pm 0.90^{b}$ & $14.13 \pm 0.91^{a}$ & $6.09 \pm 0.89^{b}$ & $<0.0001$ & $7.28 \pm 1.26^{b}$ & $10.73 \pm 0.72^{a}$ & $<0.0001$ \\
\hline Ultimate $\mathrm{pH}$ & $5.84 \pm 0.04^{b}$ & $6.05 \pm 0.05^{a}$ & $5.76 \pm 0.04^{b}$ & 0.0001 & $5.90 \pm 0.03$ & $5.87 \pm 0.07$ & 0.4278 \\
\hline Drip loss & $3.59 \pm 0.27$ & $3.76 \pm 0.36$ & $2.58 \pm 0.59$ & 0.0976 & $3.34 \pm 0.42$ & $3.28 \pm 0.35$ & 0.7821 \\
\hline Shear force $(\mathrm{N})$ & $25.33 \pm 2.37$ & $18.59 \pm 1.76$ & $24.64 \pm 2.09$ & 0.0998 & $21.86 \pm 1.94$ & $23.85 \pm 1.93$ & 0.4506 \\
\hline
\end{tabular}

a-c Superscripts on different means within a row differ significantly $(p \leq 0.05)$.

( $p=0.0007)$ followed by broiler and Aseel female and their male counterparts. Ultimate-pH of broiler breeder female meat was higher $(p=0.0133)$ as compared to male whereas broiler female had lower values. Moreover, broiler male and Aseel of both sexes showed intermediate values (Table 6).

\section{Sensory Characteristics}

Different sensory breast and thigh meat scores were obtained among broilers, broiler breeders and Aseel chickens. Aseel meat received lower scores for flavor ( $p=0.0121)$, juiciness $(p=0.0178)$ and tenderness

Table 6 - Genotype $\times$ sex interactions for meat quality attributes of Aseel chickens, broiler breeders and broilers.

\begin{tabular}{|c|c|c|c|c|c|c|c|}
\hline \multirow[t]{2}{*}{ Parameter } & \multicolumn{2}{|c|}{ Aseel } & \multicolumn{2}{|c|}{ Broiler Breeder } & \multicolumn{2}{|c|}{ Broiler } & \multirow[t]{2}{*}{$p$-value } \\
\hline & Male & Female & Male & Female & Male & Female & \\
\hline$L^{*}$ & $54.66 \pm 1.06^{b}$ & $52.79 \pm 0.83^{b}$ & $55.97 \pm 1.69^{b}$ & $55.37 \pm 1.85^{b}$ & $63.16 \pm 0.51^{a}$ & $61.79 \pm 0.67^{a}$ & $<0.0001$ \\
\hline$a^{*}$ & $10.99 \pm 0.78^{b}$ & $11.38 \pm 0.27^{b}$ & $12.84 \pm 0.22^{a}$ & $13.68 \pm 0.48^{a}$ & $9.39 \pm 0.36$ & $10.36 \pm 0.40^{\mathrm{bc}}$ & $<0.0001$ \\
\hline$b^{*}$ & $3.10 \pm 0.54^{c}$ & $8.80 \pm 0.81^{b}$ & $14.65 \pm 1.39^{a}$ & $13.61 \pm 1.25^{\mathrm{a}}$ & $3.56 \pm 0.50^{c}$ & $9.46 \pm 0.57^{b}$ & 0.0007 \\
\hline Ultimate-pH & $5.87 \pm 0.05^{b c}$ & $5.80 \pm 0.06^{b c}$ & $5.97 \pm 0.05^{b}$ & $6.12 \pm 0.06^{a}$ & $5.85 \pm 0.01^{b c}$ & $5.68 \pm 0.03^{c}$ & 0.0133 \\
\hline Drip loss & $3.54 \pm 0.30$ & $3.64 \pm 0.51$ & $3.79 \pm 0.39$ & $3.74 \pm 0.71$ & $2.70 \pm 1.24$ & $2.46 \pm 0.42$ & 0.7853 \\
\hline Shear force $(\mathrm{N})$ & $25.87 \pm 3.63$ & $24.79 \pm 3.81$ & $16.66 \pm 1.33$ & $20.52 \pm 3.16$ & $23.04 \pm 2.84$ & $26.24 \pm 3.35$ & 0.6997 \\
\hline
\end{tabular}

arc Means followed by different superscripts within a row differ significantly $(p \leq 0.05)$.

$(p=0.0477)$, indicating that the breast meat of Aseel chicken is less flavored, tender and juicy then those of broilers and broiler breeders. The panel found no color $(p=0.1886)$, aroma $(p=0.1489)$, taste $(p=0.5312)$ or acceptability $(p=0.1480)$ differences in the breast meat of the three genotypes. No significant differences between the sexes ( $p>0.05)$ were found for any breast meat sensory characteristics (Table 7). Flavor

Table 7 - Sensory evaluation of the breast meat of Aseel chickens, broiler breeders and broilers.

\begin{tabular}{|c|c|c|c|c|c|c|c|}
\hline \multirow[t]{2}{*}{ Parameter } & \multicolumn{3}{|c|}{ Genotype } & \multirow[t]{2}{*}{$p$-value } & \multicolumn{2}{|c|}{ Sex } & \multirow[t]{2}{*}{$p$-value } \\
\hline & Aseel & Broiler Breeder & Broiler & & Male & Female & \\
\hline Color & $5.60 \pm 0.22$ & $6.16 \pm 0.22$ & $5.89 \pm 0.19$ & 0.1886 & $5.82 \pm 0.19$ & $5.97 \pm 0.15$ & 0.5873 \\
\hline Aroma & $5.29 \pm 0.20$ & $5.82 \pm 0.19$ & $5.49 \pm 0.18$ & 0.1489 & $5.47 \pm 0.17$ & $5.61 \pm 0.15$ & 0.5246 \\
\hline Taste & $5.47 \pm 0.20$ & $5.82 \pm 0.23$ & $5.74 \pm 0.21$ & 0.5312 & $5.59 \pm 0.19$ & $5.78 \pm 0.17$ & 0.4515 \\
\hline Flavor & $5.33 \pm 0.21^{b}$ & $5.97 \pm 0.20^{a}$ & $6.21 \pm 0.21^{a}$ & 0.0121 & $5.72 \pm 0.18$ & $5.99 \pm 0.17$ & 0.2849 \\
\hline Juiciness & $5.23 \pm 0.24^{b}$ & $5.91 \pm 0.20^{\mathrm{a}}$ & $6.08 \pm 0.20^{a}$ & 0.0178 & $5.54 \pm 0.18$ & $5.97 \pm 0.17$ & 0.0808 \\
\hline Tenderness & $5.60 \pm 0.21^{b}$ & $5.82 \pm 0.21^{a b}$ & $6.30 \pm 0.18^{a}$ & 0.0477 & $5.85 \pm 0.16$ & $5.99 \pm 0.17$ & 0.5911 \\
\hline Acceptability & $5.54 \pm 0.22$ & $6.06 \pm 0.19$ & $6.04 \pm 0.20$ & 0.1480 & $5.75 \pm 0.17$ & $6.03 \pm 0.16$ & 0.2478 \\
\hline
\end{tabular}

a-c Means followed by different superscripts within a row differ significantly $(p \leq 0.05)$. 
( $p=0.0443)$ and juiciness ( $p=0.0267)$ values of broiler female breast were higher as compared to Aseel male whereas breast of Aseel female and Broiler breeder of both sexes showed intermediate values (Table 8).
Regarding thigh meat, Aseel scored lower for color $(p=0.0344)$ and acceptability $(p=0.0398)$ than those of broiler breeders and broilers. The panel found no difference ( $p>0.05$ ) in aroma, taste, flavor,

Table 8 - Genotype $\times$ sex interactions for breast meat organoleptic characteristics of Aseel chickens, broiler breeders and broilers.

\begin{tabular}{lccccccc}
\hline Parameter & \multicolumn{2}{c}{ Aseel } & \multicolumn{2}{c}{ Broiler Breeder } & \multicolumn{2}{c}{ Broiler } & $p$-value \\
\cline { 2 - 6 } & Male & Female & Male & Female & Male & Female \\
\hline Color & $5.56 \pm 0.35$ & $5.65 \pm 0.27$ & $5.89 \pm 0.36$ & $6.41 \pm 0.26$ & $6.00 \pm 0.28$ & $5.79 \pm 0.26$ & 0.4517 \\
Aroma & $5.03 \pm 0.28$ & $5.56 \pm 0.26$ & $5.76 \pm 0.32$ & $5.88 \pm 0.23$ & $5.61 \pm 0.25$ & $5.38 \pm 0.26$ & 0.3844 \\
Taste & $5.28 \pm 0.29$ & $5.68 \pm 0.28$ & $5.50 \pm 0.36$ & $6.12 \pm 0.30$ & $5.97 \pm 0.32$ & $5.52 \pm 0.28$ & 0.1780 \\
Flavor & $5.03 \pm 0.28^{\mathrm{b}}$ & $5.65 \pm 0.32^{\mathrm{ab}}$ & $6.00 \pm 0.28^{\mathrm{ab}}$ & $5.95 \pm 0.30^{\mathrm{ab}}$ & $6.11 \pm 0.33^{\mathrm{ab}}$ & $6.31 \pm 0.25^{\mathrm{a}}$ & 0.0443 \\
Juiciness & $4.83 \pm 0.34^{\mathrm{b}}$ & $5.65 \pm 0.33^{\mathrm{ab}}$ & $5.87 \pm 0.28^{\mathrm{ab}}$ & $5.95 \pm 0.29^{\mathrm{ab}}$ & $5.87 \pm 0.31^{\mathrm{ab}}$ & $6.26 \pm 0.25^{\mathrm{a}}$ & 0.0267 \\
Tenderness & $5.56 \pm 0.29$ & $5.65 \pm 0.32$ & $5.76 \pm 0.28$ & $5.88 \pm 0.31$ & $6.21 \pm 0.26$ & $6.38 \pm 0.25$ & 0.9900 \\
Acceptability & $5.39 \pm 0.30$ & $5.71 \pm 0.32$ & $5.84 \pm 0.28$ & $6.27 \pm 0.27$ & $6.00 \pm 0.30$ & $6.07 \pm 0.26$ & 0.8117 \\
\hline
\end{tabular}

acc Means followed by different superscripts within a row differ significantly $(p \leq 0.05)$.

juiciness and tenderness among three genotypes. Thigh meat sensory characteristics did not differ ( $p>0.05)$ between sexes (Table 9). Value of thigh color $(p=0.0505)$ was higher in broiler male than that of Aseel whereas broiler and Aseel female and broiler breeder of both sexes showed intermediate values. Aroma value $(p=0.0414)$ of broiler breeder female thigh was higher as compared to female while Broiler and Aseel showed intermediate values. Overall acceptability $(p=0.0235)$ of broiler breeder female and broiler male thigh were higher as compared to Aseel female, however, Aseel and Broiler breeder male and broiler female presented intermediate values (Table 10).

Table 9 - Sensory evaluation of the thigh meat of Aseel chickens, broiler breeders and broilers.

\begin{tabular}{|c|c|c|c|c|c|c|c|}
\hline \multirow[t]{2}{*}{ Parameter } & \multicolumn{3}{|c|}{ Genotype } & \multirow[t]{2}{*}{$p$-value } & \multicolumn{2}{|c|}{ Sex } & \multirow[t]{2}{*}{$p$-value } \\
\hline & Aseel & Broiler Breeder & Broiler & & Male & Female & \\
\hline Color & $5.15 \pm 0.22^{b}$ & $5.71 \pm 0.18^{a}$ & $5.84 \pm 0.17^{a}$ & 0.0344 & $5.52 \pm 0.17$ & $5.60 \pm 0.16$ & 0.8454 \\
\hline Aroma & $5.48 \pm 0.24$ & $5.57 \pm 0.21$ & $5.60 \pm 0.23$ & 0.9221 & $5.41 \pm 0.18$ & $5.69 \pm 0.18$ & 0.2753 \\
\hline Taste & $5.44 \pm 0.23$ & $5.44 \pm 0.19$ & $5.83 \pm 0.20$ & 0.3350 & $5.55 \pm 0.17$ & $5.57 \pm 0.18$ & 0.9484 \\
\hline Flavor & $5.48 \pm 0.23$ & $5.43 \pm 0.18$ & $5.79 \pm 0.20$ & 0.4202 & $5.57 \pm 0.15$ & $5.54 \pm 0.18$ & 0.8626 \\
\hline Juiciness & $5.32 \pm 0.22$ & $5.93 \pm 0.19$ & $5.61 \pm 0.24$ & 0.1184 & $5.69 \pm 0.16$ & $5.54 \pm 0.19$ & 0.5200 \\
\hline Tenderness & $5.48 \pm 0.22$ & $5.40 \pm 0.21$ & $5.84 \pm 0.23$ & 0.3494 & $5.50 \pm 0.17$ & $5.63 \pm 0.19$ & 0.6614 \\
\hline Acceptability & $5.42 \pm 0.22^{b}$ & $6.06 \pm 0.15^{\mathrm{a}}$ & $5.90 \pm 0.21^{\mathrm{ab}}$ & 0.0398 & $5.85 \pm 0.14$ & $5.72 \pm 0.17$ & 0.4539 \\
\hline
\end{tabular}

acc Means followed by different superscripts within a row differ significantly $(p \leq 0.05)$.

Table 10 - Genotype $\times$ sex interactions for thigh meat organoleptic characteristics of Aseel chickens, broiler breeders and broilers.

\begin{tabular}{|c|c|c|c|c|c|c|c|}
\hline \multirow[t]{2}{*}{ Parameter } & \multicolumn{2}{|c|}{ Aseel } & \multicolumn{2}{|c|}{ Broiler Breeder } & \multicolumn{2}{|c|}{ Broiler } & \multirow[t]{2}{*}{$p$-value } \\
\hline & Male & Female & Male & Female & Male & Female & \\
\hline Color & $4.98 \pm 0.31^{b}$ & $5.34 \pm 0.32^{\mathrm{ab}}$ & $5.53 \pm 0.26^{\mathrm{ab}}$ & $5.90 \pm 0.26^{\mathrm{ab}}$ & $6.14 \pm 0.26^{a}$ & $5.53 \pm 0.22^{\mathrm{ab}}$ & 0.0505 \\
\hline Aroma & $5.34 \pm 0.31^{\mathrm{ab}}$ & $5.62 \pm 0.36^{a b}$ & $4.93 \pm 0.28^{b}$ & $6.21 \pm 0.25^{a}$ & $5.97 \pm 0.33^{a b}$ & $5.24 \pm 0.29^{\mathrm{ab}}$ & 0.0414 \\
\hline Taste & $5.61 \pm 0.31$ & $5.26 \pm 0.35$ & $5.15 \pm 0.25$ & $5.73 \pm 0.28$ & $5.92 \pm 0.29$ & $5.74 \pm 0.27$ & 0.2431 \\
\hline Flavor & $5.59 \pm 0.30$ & $5.37 \pm 0.34$ & $5.30 \pm 0.24$ & $5.55 \pm 0.27$ & $5.86 \pm 0.25$ & $5.71 \pm 0.31$ & 0.6697 \\
\hline Juiciness & $5.54 \pm 0.29$ & $5.08 \pm 0.33$ & $5.83 \pm 0.22$ & $6.03 \pm 0.30$ & $5.72 \pm 0.32$ & $5.50 \pm 0.35$ & 0.5351 \\
\hline Tenderness & $5.44 \pm 0.29$ & $5.53 \pm 0.35$ & $5.00 \pm 0.28$ & $5.80 \pm 0.31$ & $6.11 \pm 0.31$ & $5.56 \pm 0.34$ & 0.1006 \\
\hline Acceptability & $5.63 \pm 0.27^{\mathrm{ab}}$ & $5.18 \pm 0.34^{b}$ & $5.73 \pm 0.21^{\mathrm{ab}}$ & $6.40 \pm 0.20^{\mathrm{a}}$ & $6.25 \pm 0.26^{a}$ & $5.53 \pm 0.32^{\mathrm{ab}}$ & 0.0235 \\
\hline
\end{tabular}

a-c Means followed by different superscripts within a row differ significantly $(p \leq 0.05)$.

\section{DISCUSSION}

In the present study broilers and broiler breeders had heavier breasts than Aseel chickens. These differences are probably due to metabolic rate differences among breeds. The findings of present study are in agreement with previous studies that reported higher $(p<0.01)$ breast yield in Hubbard and Hybro broilers compared with bare-neck, large Beladi and Betwil chickens (Tibin \& Mohamed, 1990; Hassan et al., 2006). Similarly, 
Khan U, Hussain J, Mahmud A, Khalique A, Mehmood S, Badar IH, Usman M, Jaspal MH, Ahmad S

\section{Comparative Study on Carcass Traits, Meat Quality and Taste in Broiler, Broiler Breeder and Aseel Chickens}

Nielsen et al. (2003) reported lower breast yield in slow-growing than in fast-growing chickens.

Thigh and drumstick were heavier in Aseel chickens and broiler breeders than in broilers. Aseel chickens had heavier necks followed by broiler breeders and broilers. Thigh yields mainly reflected genetic differences in breeds. Similar findings were also reported in a previous study (Rahayu et al., 2008) that obtained higher whole thigh and drumstick weights in red junglefowl than in commercial broilera. Similarly, other researchers found higher thigh meat yield in Hybro and Hubbard compared with bare-neck, large Beladi and Betwil chickens (Chhabrad \& Sapra, 1973; Hassan et al., 2006). However, Sandercock et al. (2009) reported that fast-growing broilers had higher breast and thigh meat as compared with layer or local chickens.

Liver and intestine were heavier in broilers than in broiler breeder and Aseel chickens. Furthermore, ribs and back weight was higher in broilers than Broiler Breeder, but not statistically different from Aseel. The higher liver weight in broilers may reflect excessive fat deposition and increased rate of lipogenesis. However, contradictory study also reported non-significant differences in relative weights of liver among hybrids of Cornish and Sussex, Cornish and Green-legged Partridgenous and Cobb broilers (Batkowska et al., 2015). Similarly, no significant differences were observed in liver yield among four varieties of native Aseel chickens in Pakistan (Jatoi et al., 2015). The highest heart and gizzard yields were obtained in broilers, followed by Aseel chickens, and the lowest in broiler breeders. Lower giblets weight in Broiler breeder might be attributed to the intense genetic selection for body weight, which ultimately reduced giblets weight. Similarly, significant variations were observed in heart, head and femur in naked-neck and indigenous chicken in which normal feathered chickens had higher weight than naked neck (Zein-El-Dein et al., 1981).

Female chickens showed heavier gizzards and intestines than males. It is possible that such variations in growth rate between sexes were due to the supply and demand of these organs, which underwent modifications, at least in size, to accommodate the different growth rates. Similarly, Jatoi et al. (2015) reported that male birds showed higher gizzard weight than females among four varieties of native Aseel chicken. On the other hand, another study reported that heart and liver weights did not differ between sexes among broiler strains (Plavnik \& Hurwitz, 1982).

Higher carcass, thigh, drumstick, neck and heart yields were determined in males than females. Similarly,
Rahayu et al. (2008) reported that the leg muscles of males of both red junglefowl and commercial broilers were more developed than those of females, which may be due to the physiological and behavioral differences between sexes. Moreover, Moran \& Orr (1969) also observed that the proportion of thigh and drumstick of broiler males were higher than females.

Aseel and broiler breeder meat were darker than broiler, however, broiler breeder meat was yellower and redder than those of Aseel and broiler chicken. Meat color may be influenced by the heme pigments, genetics and feeding. Similar to the present study, the breasts of chickens of an inbred Leghorn were redder than those of contemporary crossbreds (Tougan et al., 2013). Moreover, Jaturasitha et al. (2008) noted that the black-boned chickens had darker breast meat compared with other Thai Indigenous chickens. Similarly, Ponte et al. (2008) reported that the breast meat of Baetong chickens was yellower than that Praduhangdum and black-boned chickens. Lonergan et al. (2003) reported that difference in redness among different genotypes was due to differences in muscle fiber type.

In the present study, the breast meat of females was yellower and redder compared to that of males. This result is consistent with the finding of Tougan et al. (2013), who reported that female broilers exhibited a higher yellowness $\left(b^{*}\right)$ value than males.

Meat ultimate-pH value was lower in broilers and Aseel chickens that in broiler breeders. These $\mathrm{pH}$ differences are probably due to the differences in muscle type and glycogen content, which change according to the proportion of the muscle fibers that are responsible for different patterns of muscle metabolism. The findings of present study are in line with another study who found significant differences between the ultimate $\mathrm{pH}$ among different genotypes of chicken (Xiong et al., 1993; Fernandez et al., 2002). Similarly, Debut et al. (2003) reported that the rate of $\mathrm{pH}$ decline of slow-growing chicken lines is faster than in fast growing lines. However, the study of Youssao et al. (2012), carried out in Benin, evaluated Label Rouge and indigenous chickens of North and South ecotypes and reported no $\mathrm{pH}$ differences recorded after 1 and 24 hours post slaughtering among genotypes.

Regarding sensory evaluation, the breast meat of Aseel chicken received lower flavor, tenderness and juiciness scores than those of broiler and broiler breeder. Better flavor of broiler breast might be due to increased fat levels in the meat, however, the differences in juiciness might be attributed to higher 
Khan U, Hussain J, Mahmud A, Khalique A, Mehmood S, Badar IH, Usman M, Jaspal MH, Ahmad S

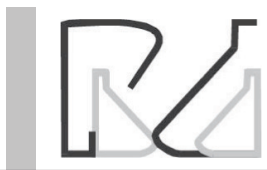

Comparative Study on Carcass Traits, Meat Quality and Taste in Broiler, Broiler Breeder and Aseel Chickens content of water and intramuscular fat. Regarding thigh meat, Aseel scored lower for color and acceptability than broiler breeders and broilers. Thigh meat color may be influenced by species, diet, type of muscle fiber and exercise. The sensory results obtained are not consistent with the findings of Huang et al. (2007) and Jayasena et al. (2013) reported that the unique flavors of native chickens are preferred in Chinese or Korean cuisine. Moreover, Bogosavljević-Boškivić et al. (2010) reported that semi-intensive rearing systems help produce products with better flavor compared with conventionally-produced broiler chickens. However, another study reported non-significant variation among different chicken genotypes regarding appearance and flavor (Rajkumar et al., 2016). The obtained juiciness results are in agreement with Amorim et al. (2016), who reported that broilers scored higher juiciness score compared with Amarela roosters. Moreover, Breast meat of broiler chickens reared on intensive system showed better juiciness (6.55) compared with those on semi-intensive system (5.55) (Olaifa et al., 2016).

\section{CONCLUSIONS}

It is concluded that variation exists among different breeds of chickens and their sexes. Carcass traits of broilers were comparable with those of Aseel chickens; however, broiler breeders showed better meat quality traits. Broiler and broiler breeder meat scored higher for sensory evaluation. Male birds showed better carcass and meat quality traits than females.

\section{ACKNOWLEDGEMENTS}

Authors gratefully acknowledge the administration at Department of Poultry Production, Department of Meat Science and Technology and Department of Animal Nutrition, University of Veterinary and Animal Sciences, Lahore, Pakistan.

\section{DISCLOSURE STATEMENT}

No potential conflict of interest was reported by the authors.

\section{REFERENCES}

Amorim A, Rodrigues S, Pereira E, Teixeira A. Physicochemical composition and sensory quality evaluation of capon and rooster meat. Poultry Science 2016; 95: 1211-1219.

Bell DD, Weaver WD. Pages 1124-1125 in Commercial chicken meat and egg production, M.A. Norwell, Kluwer Academic. 2002.
Batkowska J, Brodacki A, Zieba G, Horbanczuk JO, Lukaszewicz M. Growth performance, carcass traits and physical properties of chicken meat as affected by genotype and production system. Archives Animal Breeding 2015; 58: 325-333.

Bogosavljevic-Boskovic S, Mitrovic S, Djokovic R, Doskovic V, Djermanovic $V$. Chemical composition of chicken meat produced in extensive indoor and free range rearing systems. African Journal of Biotechnology 2010; 27: 9069-9075.

Castellini C, Mugnai C, Dal Bosco A. Effect of organic production system on broiler carcass and meat quality. Meat Science 2002; 60: 219-225.

Chhbardra AD, Sapra KL. Growth, mortality and carcass quality traits of indigenous and exotic pure-breeds and their crosses. Indian Veterinary Journal 1973; 50: 1-7.

Debut M, Berri C, Baeza E, Sellier N, Arnould C, Guemene D, Jehl N, Boutten B, Jego Y, Beamont C, Le Bihan-Duval E. Variation of chicken technological meat quality in relation to genotype and pre slaughter stress conditions. Poultry Science 2003; 82: 1829-1838.

Economic Survey. Pakistan Economic Survey, Pages 41-42 in Chap 2, Agriculture, III. Livestock and Poultry, 2016-17

Fernandez X, Santé V, Baeza E, Lebihan-Duval E, Berri C, Rémignon H, Babilé $\mathrm{R}$, Le Pottier $\mathrm{G}$, Astruc T. Effects of the rate of muscle post mortem $\mathrm{pH}$ fall on the technological quality of turkey meat. British Poultry Science 2002; 43: 245-252.

Hassan H, Neser FWC, Tadelle D, Evan MK. Studies on the growth performance of native chicken ecotypes and RIR chicken under improved management system. In North West Ethiopia. Livestock. Research and Rural Development 2006; 18: 76.

Honikel KO. How to measure the water-holding capacity of meat? Recommendation of a standardized method. Pages 129-142 in Evaluation and Control of Meat Quality in Pigs. The Hague, Netherlands, 1987

Huang CC, Hsieh CC, Chiang SH. Estimating the energy partitioning of Taiwanese native chickens by mathematical model. Animal Feed Science and Technology 2007; 134: 189-197.

Jatoi AS, Iqbal M, Sahota AW, Akram M, Javed K, Mehmood S, Hussain J, Ishaq HM. Carcass characteristics and organ development in four different varieties of native Aseel chicken of Pakistan. Pakistan Journal of Science 2015; 67: 127-132.

Jaturasitha S, Leangwunta V, Leotaragul A, Phongphaew A, Apichartsrungkoon T, Simasathitkul N, Vearasilp T, Worachai L, Meulen $U$. A comparative study of Thai native chicken and broiler on productive performance, carcass and meat quality. Deutscher Tropentag, Page 146 in Challenges to Organic Farming and Sustainable Land Use in the Tropics and Subtropics. Book of Abstracts. Univ Kassel, Germany, Witzenhausen, 2002

Jaturasitha S, Srikanchai T, Kreuzer M, Wicke M. Differences in carcass and meat characteristics between chicken indigenous to northern Thailand (Black-boned and Thai native) and imported extensive breeds (Bresse and Rhode Island Red). Poultry Science 2008; 87: 160-169.

Jayasena DD, Jung S, Kim HJ, Bae YS, Yong HI, Lee JH, Kim JG, Jo C. Composition of quality traits of meat from Korean native chicken and broilers British Poultry Science used in two different traditional Korean cuisines. Asian-Australasian Journal of Animal Science 2013; 26: 10381046.

Karakök SG, Ozogul Y, Saler M, Ozogul F. Proximate analysis. Fatty acid profiles and mineral contents of meats. Journal of Muscle Foods 2008; 21: 210-223. 
Khan U, Hussain J, Mahmud A, Khalique A, Mehmood S, Badar IH, Usman M, Jaspal MH, Ahmad S

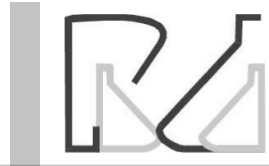

Klont RE, Brocks L, Eikelenboom G. Muscle fibre type and meat quality. Meat Science 1998; 49: 219-229.

Lonergan SM, Deeb N, Fedlet CA, Lamont SJ. Breast meat quality and composition in unique chicken populations. Poultry Science 2003; 82: 1990-1994.

Memon NA. Pages 27-30 in Poultry: Country's second-largest industry. Exclusive on Poultry, 2012.

Moran ET, Orr HL. A characterization of the chicken broiler as a function of sex and age: Live performance, processing, grade and cooking yields. Food Technology 1969; 23: 1077-1084.

Nielsen BL, Thomsen MG, Rensen PS, Young JF. Feed and strain effects on the use of outdoor areas by broilers. British Poultry Science 2003; 44: 161-169.

Olaifa RO, Sogunle OM, Okubanjo AO. Evaluation of Quality, Organoleptic Attributes and Chemical Composition of Broiler Chicken Meat Reared on Intensive and Semi-Intensive Systems. Journal of Meat Science and Technology 2016; 4: 25-32.

Pearson AM, Gillet TA. Reduced and low-fat meat products. Processed Meats. Chapman and Hall, New York, 1996.

Plavnik I, Hurwitz S. Organ weights and body composition in chickens as related to the energy and amino acid requirements: Effects of strain, sex and age. Poultry Science 1982; 62: 152-163.

Ponte PIP, Prates JAM, Crespo JP, Crespo DG, Mourao JL, Alves SP, Fontes CMGA. Restricting the intake of a cereal-based feed in free-rangepastured poultry: Effects on performance and meat quality. Poultry Science 2008; 87: 2032-2042.

PPA. An Overview of Pakistan Poultry Industry. Available at: http:// pakistanpoultry.org/index.php/poultry-industries/an-overview-ofpoultry-industry/. Accessed on July 28, 2016.

Rahayu HSI, Zulkifli I, Vidyadaran MK, Alimon AR, Babjee SA. Carcass Variables and Chemical Composition of Commercial Broiler Chickens and the Red Jungle Fowl. Asian-Australasian Journal of Animal Sciences 2008; 21: 1376-1382.

Rajkumar U, Muthukumar M, Haunshi S, Niranjan M, Raju MVLN, Rama Rao SV, Chatterjee RN. Comparative evaluation of carcass traits and meat quality in native Aseel chickens and commercial broilers. British Poultry Science 2016; 57: 339-347.

\section{Comparative Study on Carcass Traits, Meat Quality and Taste in Broiler, Broiler Breeder and Aseel Chickens}

Sandercock DA, Nute GR, Hocking PM. Quantifying the effects of genetic selection and genetic variation for body size, carcass composition, and meat quality in the domestic fowl (Gallus domesticus). Poultry Science 2009; 88: 923-931.

SAS Institute. SAS® Users Guide: Statistics. Version 9.01.SAS Institute Inc., Cary, N.C. 2002-2004.

Shahzad M, Rizvi F, Khan A, Siddique M, Khan MZ, Bukhari SM. Diagnosis of Avian Paramyxovirus type-1 infection in chicken by immunoflourescence technique. International Journal of Agriculture Biology 2011; 13: 266-270.

Stadig LM, Rodenburg TB, Reubens B, Aerts J, Duquenne B, Tuyttens FAM. Effects of free- range access on production parameters and meat quality, composition and taste in slow growing broiler chickens. Poultry Science 2016; 95: 2971-2978.

Tibin IM, Mohamed KA. The effect of breed and dietary protein level on the performance and carcass characteristics of exotic meat- type strain and Sudanese native chickens (1) relationship of feed intake, live weight, feed conversion ratio and mortality with breed and dietary protein level. Sudan Journal of Veterinary Science and Animal Husbandry 1990; 29: $13-23$.

Tougan PU, Dahouda M, Salifou CFA, Ahounou SGAT, Kpodekon M Mensah GA, Thewis A, Karim IYA. Conversion of chicken muscle to meat and factor affecting chicken meat quality: a review. International Journal of Agronomy and Agricultural Research 2013; 3: 1-20.

Xiong XL, Cantor AH, Pescatore AJ, Blanchard SP, Straw ML. Variations in muscle chemical composition, $\mathrm{pH}$, and protein extractability among different broiler crosses. Poultry Science 1993; 72: 583-588.

Young LL, Northcutt JK, Buhr RJ, Lyon CE, Ware GO. Effects of age, sex, and duration of postmortem aging on percentage yield of parts from broiler chicken carcasses. Poultry Science 2001; 80: 376-379.

Youssao IAK, Alkoiret IT, Dahouda M, Assogba1 MN., Idrissou ND, Kayang BB, Yapi-Gnaoré V, Assogba HM, Houinsou AS, Ahounou SG, Tougan UP, Rognon X, Tixier-Boichard M. Comparison of growth performance, carcass characteristics and meat quality of Benin indigenous chickens and Label Rouge (T55×SA51). African Journal of Biotechnology 2012; 11: 15569-15579

Zein-El-Dein A, Zeiny M, Ayoub H. Carcass measurement of naked neck and normal chicks. Annales de génétique et de sélection animale 1981; 13: $435-440$. 
